Research Paper:

\title{
Effect of Assertiveness Training on Adaptive and Aggressive Assertiveness in Nurses
}

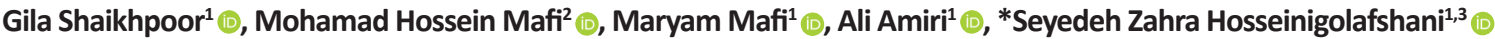

1. Department of Critical Care Nursing, School of Nursing and Midwifery, Qazvin University of Medical Science, Qazvin, Iran.

2. Student Research Committee, Mashhad University of Medical Sciences, Mashhad, Iran.

3. Social Determinants of Health Research Center, Research Institute for Prevention of Non-Communicable Diseases, Qazvin University of Medical Sciences, Qazvin, Iran.

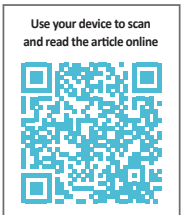

Citation Shaikhpoor G, Mafi MM, Mafi M, Amiri A, Hosseinigolafshani SZ. [Effect of Assertiveness Training on Adaptive and Aggressive Assertiveness in Nurses (Persian)]. Journal of Inflammatory Diseases. 2020; 24(3):212-223. https://doi.org/10.32598/JQUMS.24.3.2

dei https://doi.org/10.32598/JQUMS.24.3.2

Received: 28 Dec 2019 Accepted: 16 Jun 2020 Available Online: 01 Aug 2020

Keywords:

Assertiveness, Adaptive assertiveness, Aggressive assertiveness, Nurses

\section{A B S T R A C T}

Background Assertiveness is one of the most important skills needed by nurses to reduce interpersonal conflict and build an effective teamwork in complex hospital environments.

Objective The aim of this study was to determine the effect of an assertiveness training program on nurses' aggressive and adaptive assertiveness.

Methods In this interventional study, participants were 64 nurses working in hospitals of Abhar city in Iran, randomly divided into two groups of intervention $(n=32)$ and control $(n=32)$. Assertive training program was presented as a two-day training workshop (10 hours) to the intervention group, while the control group received no training. Thompson and Burnham's Adaptive and Aggressive Assertiveness Scale were completed by the participants in both groups at baseline, immediately, one and three months after the intervention. After collecting the data, Friedman, Mann-Whitney, Chi-squared, and Fisher tests were used for their analysis.

Findings The mean of adaptive assertiveness score was significantly different between the the two groups immediately $(P=0.002)$ and 1 month after the intervention $(P=0.025)$. The aggressive assertiveness scores were not significantly different immediately, 1 and 3 months after the intervention $(P>0.05)$, but the results of Friedman test showed a statistically significant decrease in the aggressive assertiveness scores compared to its baseline score $(P=0.002)$.

Conclusion The assertiveness training program increased adaptive assertiveness and decreased aggressive assertiveness in nurses. Relevant authorities can design continuous and useful assertiveness training programs to promot this valuable skill in nurses.

\section{Extended Abstract}

\section{Introduction}

roper relation is the main influential factor in the nursing profession [3]. One of important components in human relations is the assertiveness skill [4]. Assertiveness is generally defined as the expression of personal needs, rights and feelings without denying or violating the rights of others [3]. Nowadays, assertiveness is an unavoidable skill and necessity in the nurs-

* Corresponding Author:

Seyedeh Zahra Hosseinigolafshani

Address: Social Determinants of Health Research Center, Research Institute for Prevention of Non-Communicable Diseases, Qazvin University of Medical Sciences, Qazvin, Iran.

Tel: +98 (916) 8439285

E-Mail: z.hoseinigolafshani@qums.ac.ir 
ing profession [12]. Because of the complicated interpersonal relations of a nurse with other treatment team members, patients and their families, and also due to occupational challenges of people with this career, many studies have claimed assertiveness as a fundamental skill $[13,14]$. One of ways to gain or improve assertiveness is to train it in a professional way [16]

The results of studies have demonstrated that assertiveness training can result in increased assertiveness and assertive interactions in the workplace $[16,18,19,21]$. The important issue is the difference between two concepts of adaptive assertiveness and aggressive assertiveness. Aggressive assertiveness is a destructive act and can result in numerous negative consequences, while adaptive assertiveness is a positive act that strengthens daily interactions [7]. The present study was performed with aim of studying the effect of assertiveness training on adaptive and aggressive assertiveness in nurses.

\section{Materials and Methods}

This is an interventional study with two experimental and control groups. Study population consists of all nurses working in Alghadir, Emdadi and Omid hospitals in Abhar, Iran. A sample size of 64 was determined according to Motahari et al. [9] and considering Type I error $\alpha=0.05$ and Type II error $\beta=0.01$. At first, 32 nurses in each group entered the study. At the end, 6 nurses were excluded due to their absence, and 1 nurse in control group refused to continue participation. Both groups filled out the Adaptive and Aggressive Assertiveness Scale developed by Thompson and Berenbaum [11] at baseline. Then, the training program was presented to the nurses in experimental group for 2 days (10 hours). The control group received no intervention but a training package was provided to them at the end of intervention to observe ethical principles. Immedi- ately, 1 and 3 months after intervention, assertiveness was re-evaluated in both groups. The hypotheses were tested at a significance level of less than 0.05 in SPSS v. 24.

\section{Results}

The results showed the mean score of adaptive assertiveness was not significantly different at baseline between the two groups $(\mathrm{P}=0.090)$, but immediately after intervention, a significant difference was reported $(\mathrm{P}=0.002)$. One month after intervention, the mean score of adaptive assertiveness was significantly different between the two groups $(\mathrm{P}=0.025)$; but the difference was not significant 3 months after intervention $(\mathrm{P}=0.136)$. The mean score of aggressive assertiveness at baseline $(\mathrm{P}=0.53)$, immediately after intervention $(\mathrm{P}=0.16), 1$ month after intervention $(\mathrm{P}=0.44)$ and 3 months after intervention ( $\mathrm{P}=0.43$ ) showed no significant difference between the two groups (Table 1).

\section{Discusion}

Based on the results, the assertiveness training program improved adaptive assertiveness in nurses, but its effect was not stable such that, after three months, their skill was declined. It can be concluded that the assertiveness training could somehow affect the attitudes of nurses and led to increased adaptive assertiveness in them, but decreased score of adaptive assertiveness and increased score of aggressive assertiveness 3 months after intervention probably was due to the lack of a clear strategy for continuation of training principles. Results of Motahari et al. also reported the effectiveness of assertiveness training in increasing nurses' assertiveness [9].

In previous studies, the results have shown the positive effect of training programs on increasing assertiveness, but their difference with the present study is related to the differentiation of assertiveness types. In other studies, the as-

Table 1. Comparing the Mean \pm SD scores of aggressive assertiveness before and after intervention

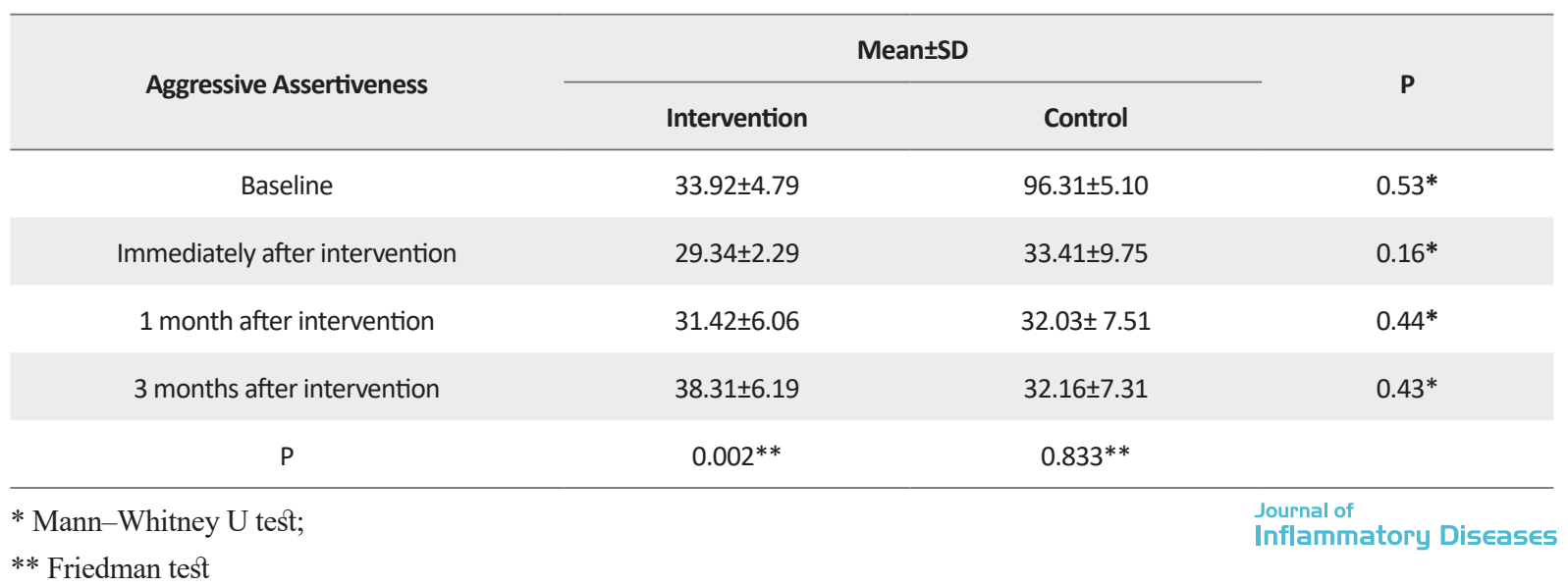


sertiveness has been reported with an overall score, while in the present study assertiveness was divided into two types of aggressive and adaptive. In our study, assertiveness training could affect aggressive assertiveness, but the mean score showed no significant difference at three time spans in comparison with the control group. In Motahari et al.'s study [9], intra-group differences were reported, similar to our study in terms of aggressive assertiveness, but the score was not reported based on the type of assertiveness.

\section{Ethical Considerations}

Compliance with ethical guidelines

This study obtained its ethical approval fron the Ethic Committee of Qazvin University of Medical Sciences (Code: IR.QUMS.REC.1397.263).

Funding

This study was extracted from the master thesis of first author Department of Critical Care Nursing, School of Nursing and Midwifery, Qazvin University of Medical Science.

\section{Authors' contributions}

Conceptualization and initial draft preparation: Gila Shakhpoor and Seyedeh Zahra Hosseinigolafshani; Data collection and writing: Mohammad Hossein Mafi; Data analysis: Maryam Mafi; Intervention and writing: Ali Amiri.

\section{Conflicts of interest}

The authors declare no conflict of interest.

\section{Acknowledgements}

The authors would like to appreciate the authorities at the department of nursing, Qazvin University of Medical Sciences. Also, we thanks of all nurses who participated in this study. 


\title{
بررسى تأثير آموزش جرئتورزى بر جسارت سازگًارانه و برخاشًرانه يرستاران
}

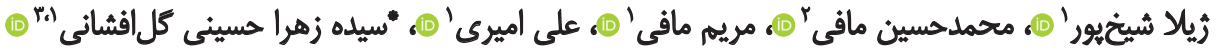

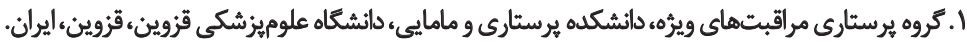

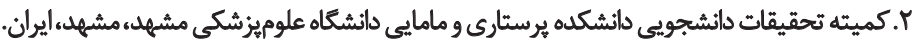

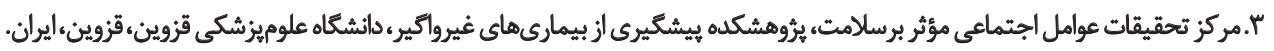

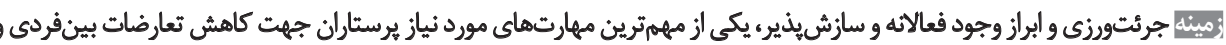

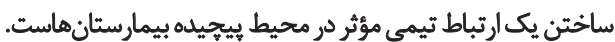

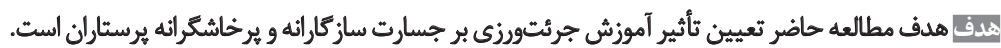

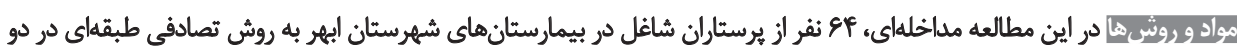

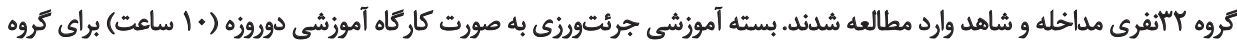

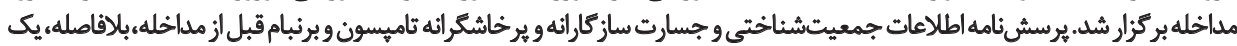

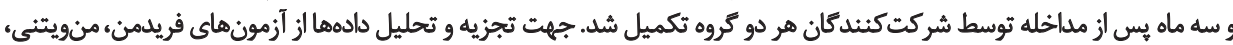
كائ ودو وفيشر استفاده شد.

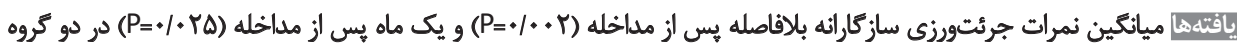

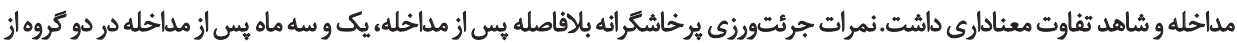

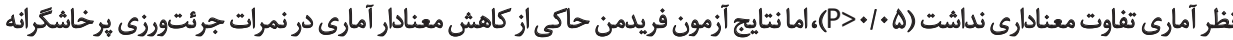

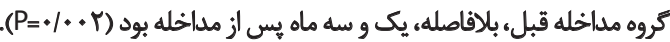

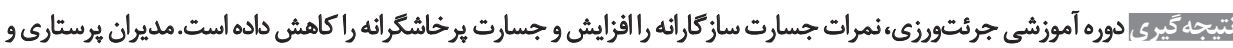

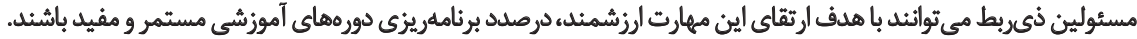

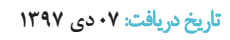

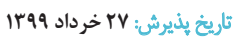
تاريخ اتتشار: المرداد

و رضايت مددجويان، تأثير قابل توجهى نيز بر سرآهدى تيم

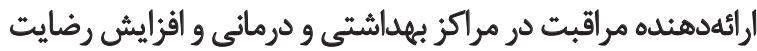

مقeم

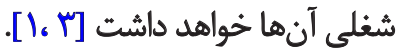

امروزه سيستمهاى بهداشت و درمان به دليل ارتباط مستقيم

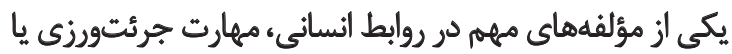

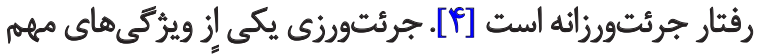

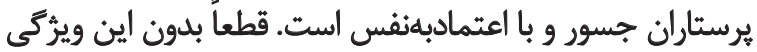

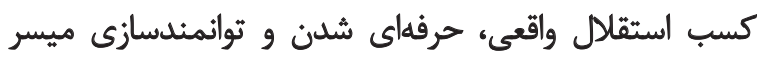

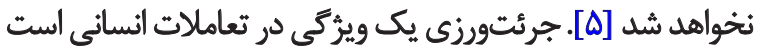

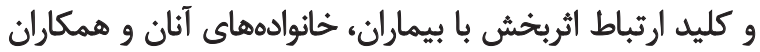

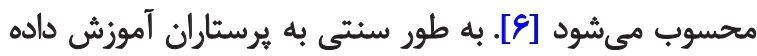

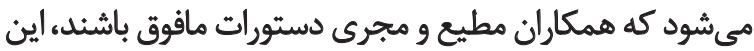

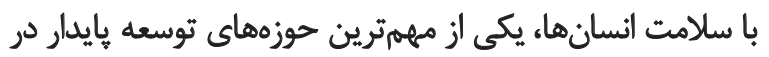

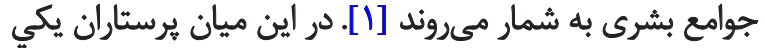

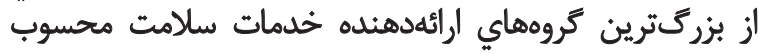

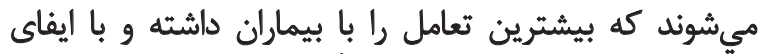

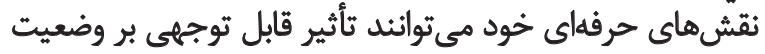

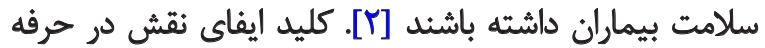

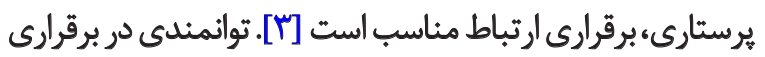
ارتباطات قوى و اثربخش، علاوه بر تضمين كيفيت مراقبتها 
كيم" و همكاران (Y. (Y) شاخصهاى جرئتورزى، صلاحيت

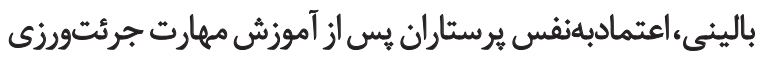

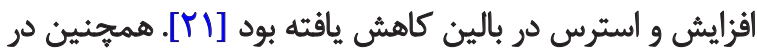

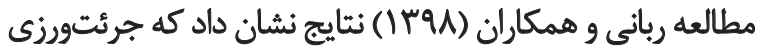

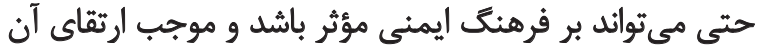

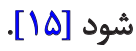

نتايج مطالعات متعدد نشان داد كه آموزش جرئتورزى ميىتواند

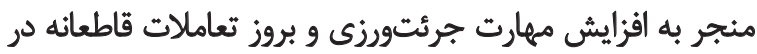

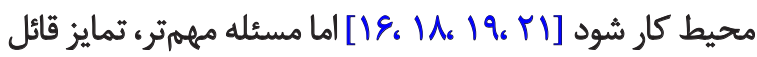

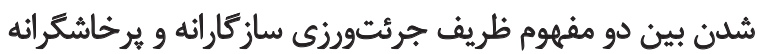

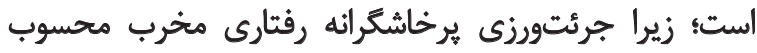

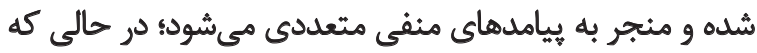

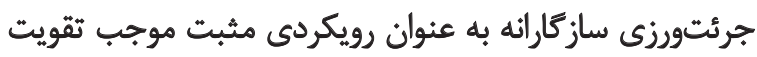

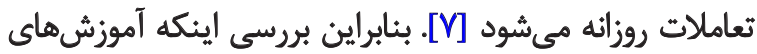

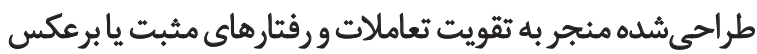

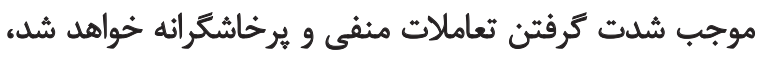

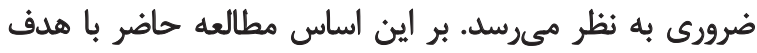

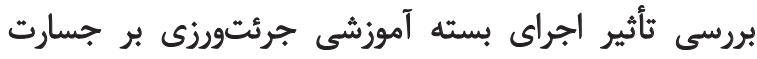

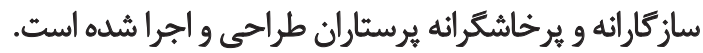

\section{llo}

مطالعه حاضر از نوع يروهش هاى مداخلهايى باطراحى دو تروه

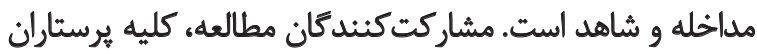

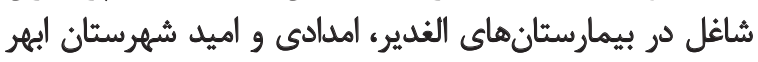

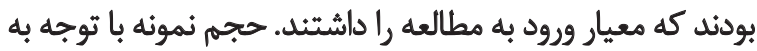

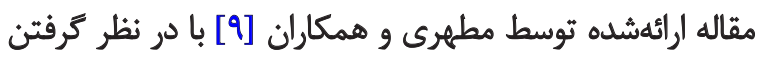

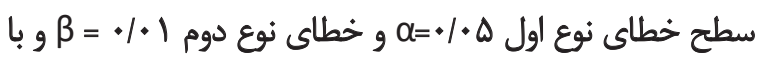

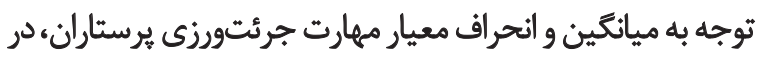

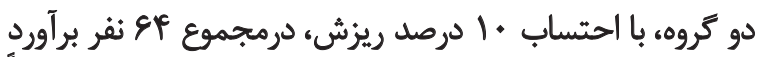

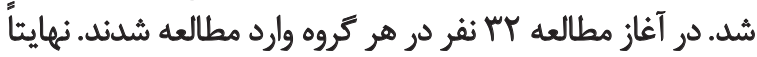

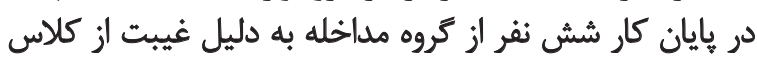

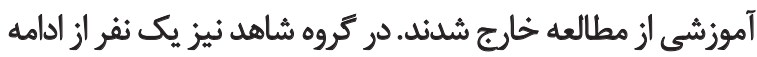

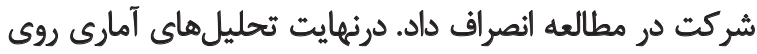
ابت نفر از تروه شاهد و عب نفر از تروه مداخله انجام شد.

معيارهاي ورود به مطالعه شامل حداقل سابقه شش مان ماه كار

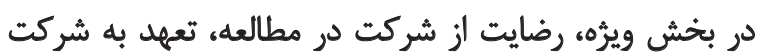

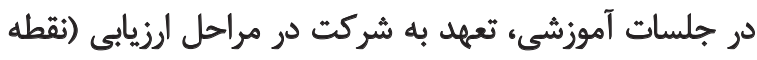

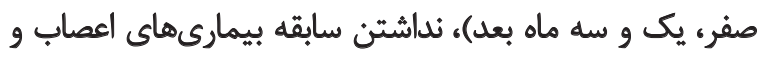

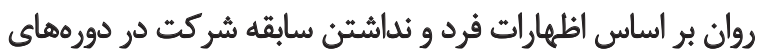

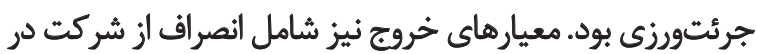

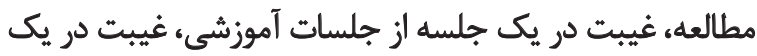
جلسه از مراحل ارزيابي سهُ دئه بوده

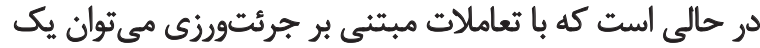

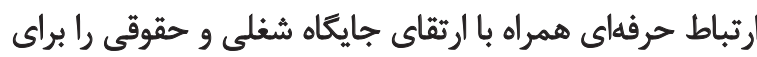

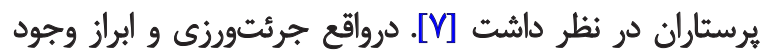

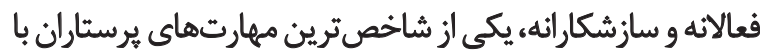

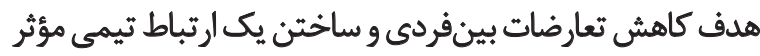

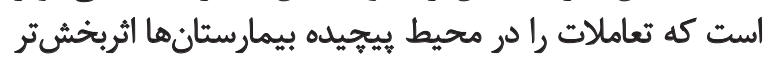

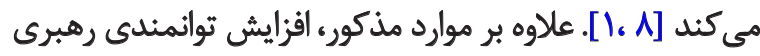

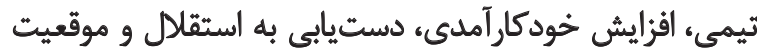

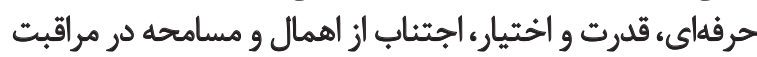

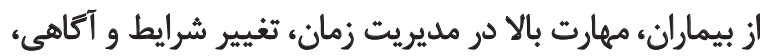

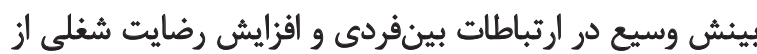

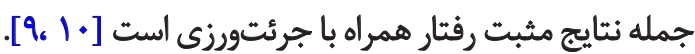

جرئتورزى به طور معمول با بيان صادقانه و مشروعيت آراء

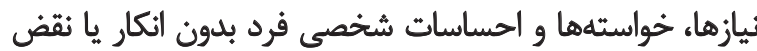

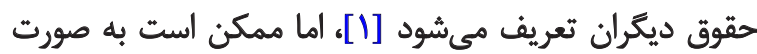

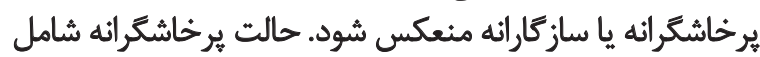

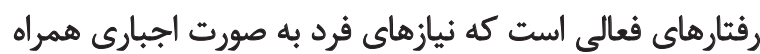

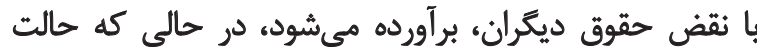

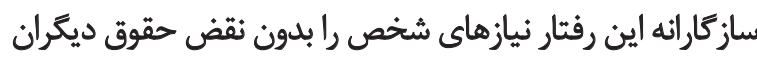

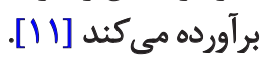

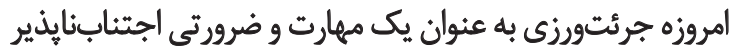

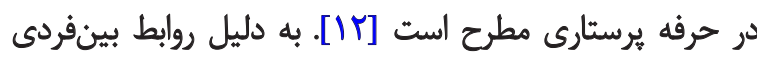

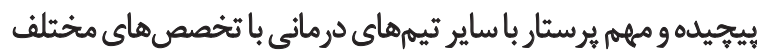

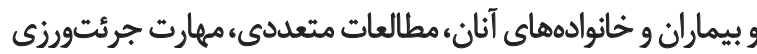

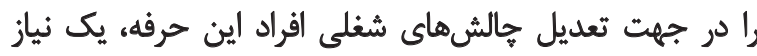

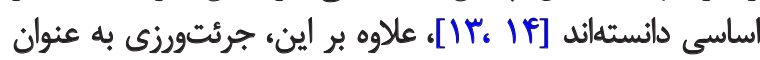

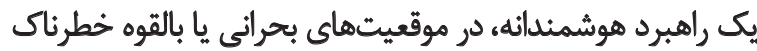

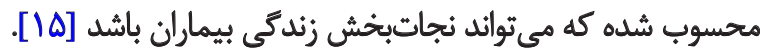

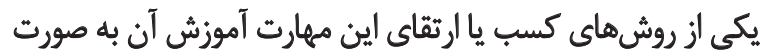

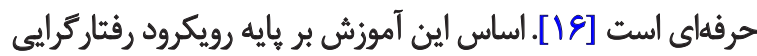

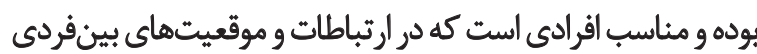

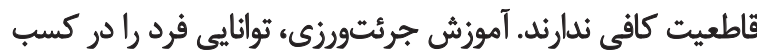

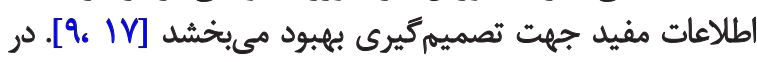

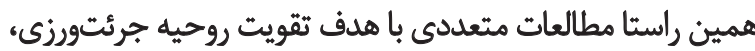
طراحى و اجرا شده است. نتايج اغلب اين مطالعات نشان منان داده است

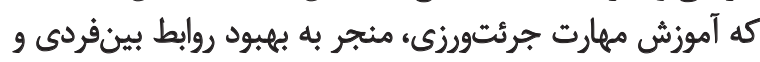

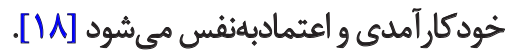

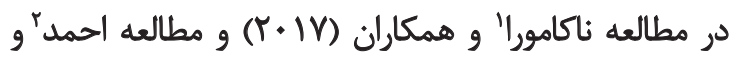

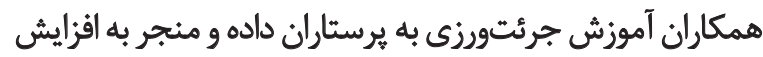

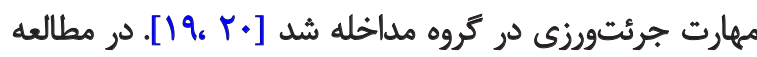

1. Nakamura

2. Ahmed 
يرسش نامه مطرح شده است. مشاركت كنيدكًان يُروهش ميزان

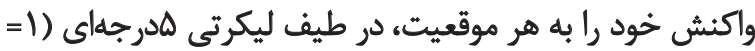

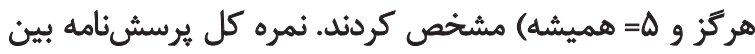

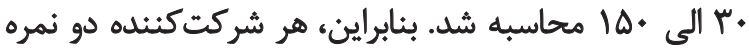

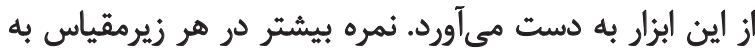

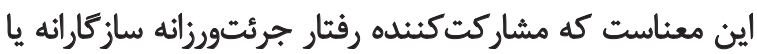

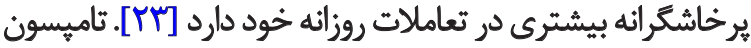

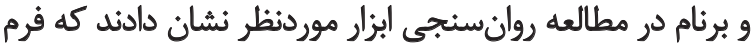

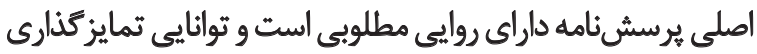

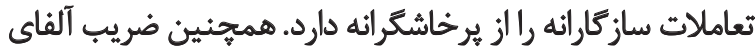

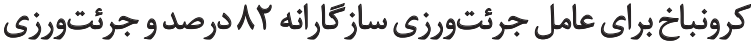

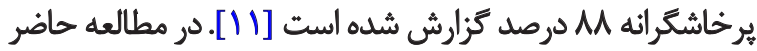

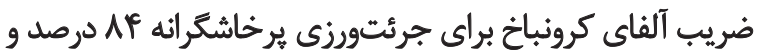

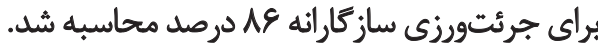

جهت بررسى دادها از آمارههاى توصيفى، فراوانى و درصد

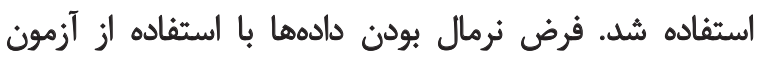

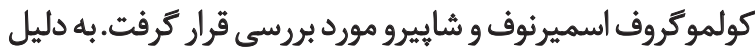

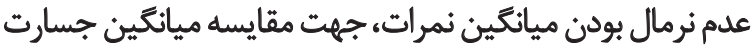

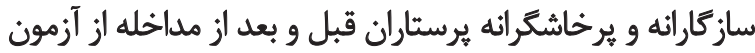

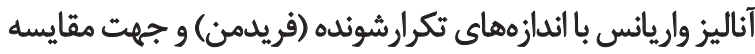

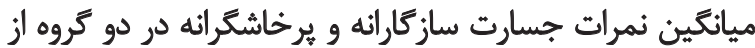

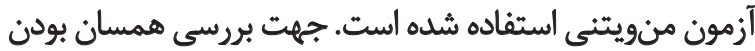

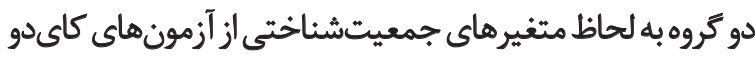

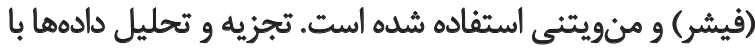

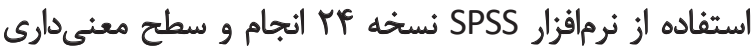
كمتر از ه • اء در نظر ترفته شد.

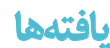

نتايج اطلاعات هV هيرستار در گروه مداخله و شاهد مورد

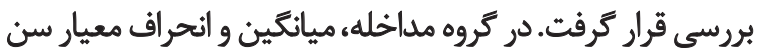

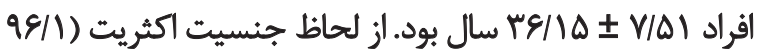

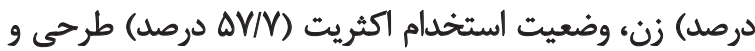

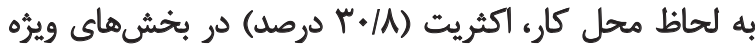

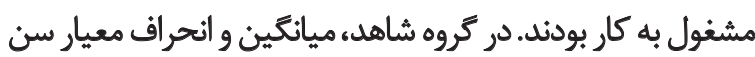

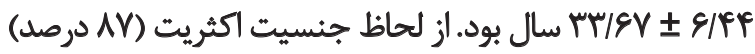

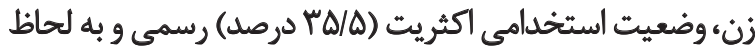

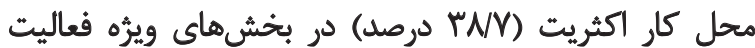

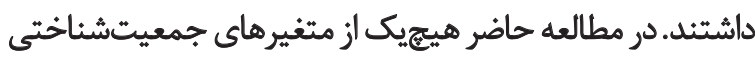

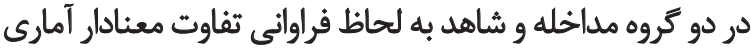

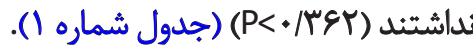
در خصوص ميانكين نمرات جرئتورزى، نتايج مطالعه نشان

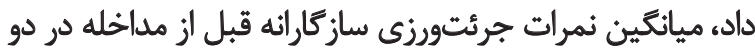

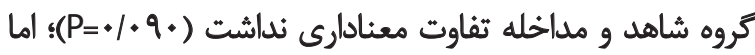

جهت انتخاب مشاركت كثندكان،در ابتدا ليست كليه يرستاران

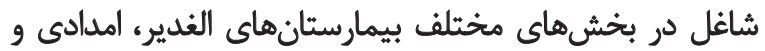

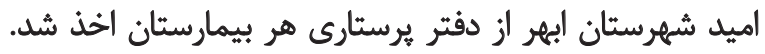

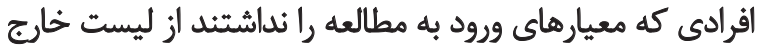

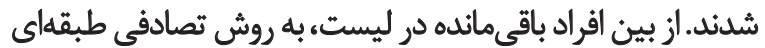

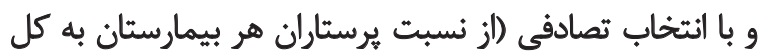

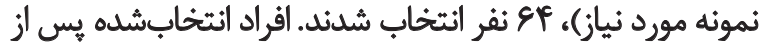

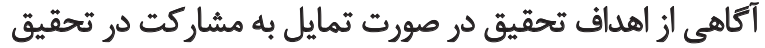

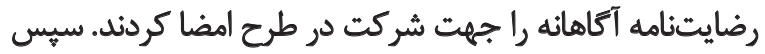

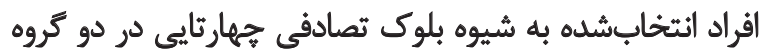

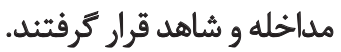

هر دو گروه قبل از مداخله برسشنامه جرئتورزى را تكميل

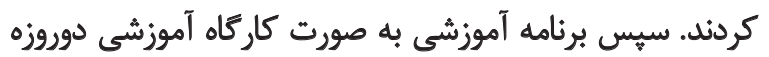

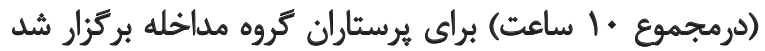

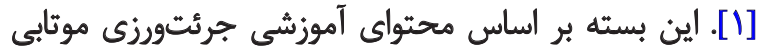

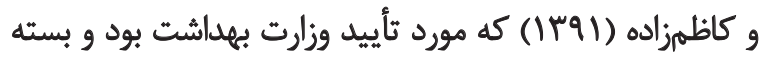

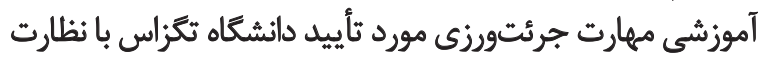

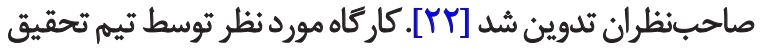

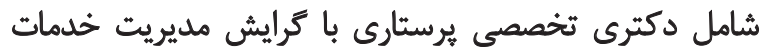

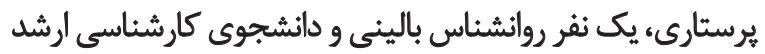

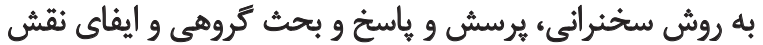

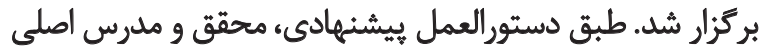

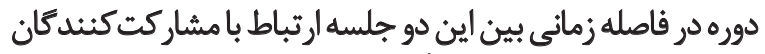

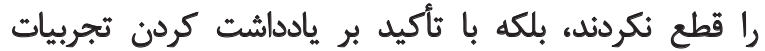

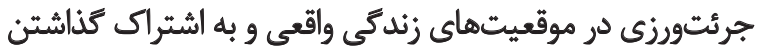

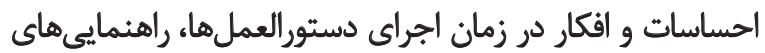

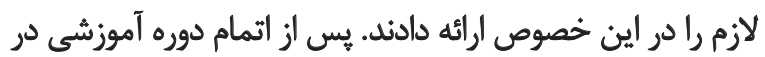

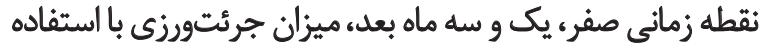

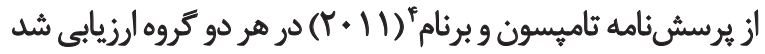

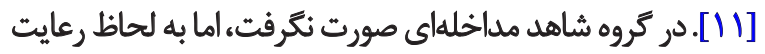

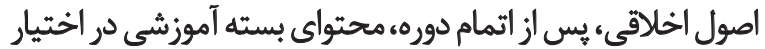

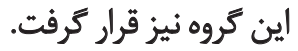

ابزارهاي استفادشده در اين مطالعه برسشنامه اطلاعات

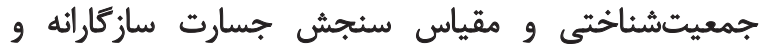

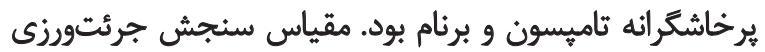

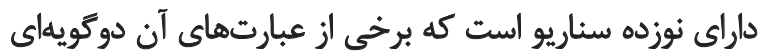

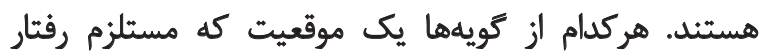

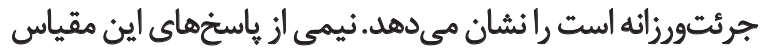

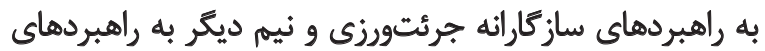

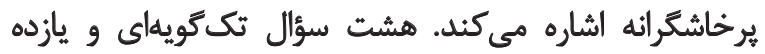

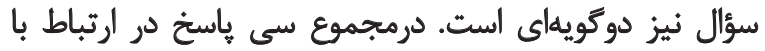

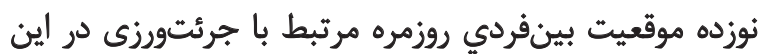


جدول ا. مشخصات جمعيتشئاختى واحدهاى مورد يثوهش در دو تروه مداخله و شاهد

\begin{tabular}{|c|c|c|c|c|}
\hline \multirow{2}{*}{ أزمون آمارى (كاي اسكوثر) } & شاهد & مداخله & \multirow{2}{*}{ كروه سطوح } & \multirow{2}{*}{ مثغير } \\
\hline & تعداد (درصد) & تعداد (درصد) & & \\
\hline \multirow{2}{*}{$P=. / M Q T$} & $r V(A V)$ & $r \Delta(q S N)$ & زن - ن & \multirow{2}{*}{ جنسيت } \\
\hline & $r(I T)$ & $1(r / 9)$ & هرد & \\
\hline \multirow{2}{*}{$P=* / \Delta \cdot \Lambda$} & $\Delta(19 / 1)$ & $9(\pi T / 1)$ & مجرد & \multirow{2}{*}{ ت تأهل } \\
\hline & $r \&(A r / q)$ & $r+(N \& / q)$ & متاهل & \\
\hline \multirow{2}{*}{$P=+/ 9 Y$} & $r(q \cdot / r)$ & $M(q Y / \pi)$ & ليسائس & \multirow{2}{*}{ تحصيلات } \\
\hline & $r(q / V)$ & $r(N M)$ & فوق ليسانس & \\
\hline \multirow{5}{*}{$P=* / 1 \cdot 1$} & $r(1 r / 9)$ & $r(11 / \Delta)$ & قراردادى & \multirow{5}{*}{ استخدام } \\
\hline & $\|(r \Delta / \Delta)$ & $r(11 / \Delta)$ & رسمى & \\
\hline & $\Lambda(T / A)$ & IQ $(\Delta \vee M)$ & طرحى & \\
\hline & $\Delta(\mid g / 1)$ & $P(1 \Delta / \Delta)$ & شركتى & \\
\hline & $r(q / V)$ & $1(r / A)$ & ييمانى & \\
\hline \multirow{7}{*}{$P \equiv * 1.91$} & $r(q / V)$ & $f(T)$ & إخلى & \multirow{7}{*}{ بخش محل كار } \\
\hline & $r(1 r / 9)$ & $r(V / E)$ & كودكان & \\
\hline & $M(T N V)$ & $\Lambda(\mu \circ / \Lambda)$ & ويزه & \\
\hline & $\cdot(\cdot)$ & $r(N / P)$ & درمائكاه تُخحصصى & \\
\hline & $r(q / V)$ & $f\left(1 \otimes / r^{q}\right)$ & اثأ عمل / اجراحى & \\
\hline & $\Lambda(Y \Delta / A)$ & $I(r / A)$ & اورزأس & \\
\hline & $I(T / M)$ & $r(11 / \Delta)$ & اعصاب و ووان & \\
\hline نتيجه أزمون منويتنى & شاهد & مداخله & \multicolumn{2}{|c|}{ متغيرهاى كمى مطالعه } \\
\hline$P \equiv=/ 194$ & $M / e V \pm E / M P$ & $r / \mid \Delta \pm V / \Delta 1$ & \multicolumn{2}{|c|}{ سن يبرسثاران (سال) } \\
\hline $\mathrm{P}=\cdot /$ MAT & 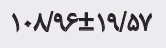 & $\Lambda_{*} / \mathrm{N} \pm 1 \otimes / A T$ & \multicolumn{2}{|c|}{ سابقه كار (ماه) } \\
\hline
\end{tabular}

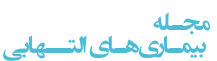

ماه بعد از مداخله اختلاف معنادارى وجود داشت (Y + • P= P)؛ در

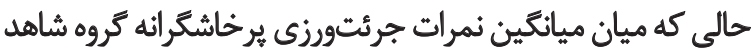

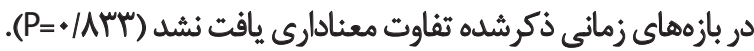

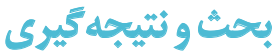

در مطالعه حاضر، ميانغين نمرات جرئتورزى سازكارانه

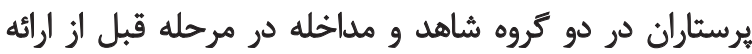

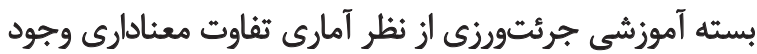

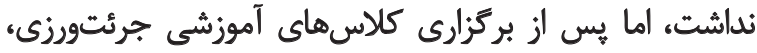

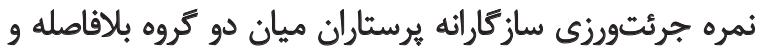

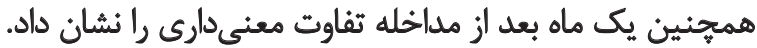

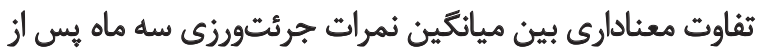

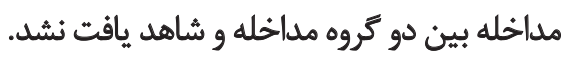

بلافاصله بعد از مداخله، ميان دو كروه مداخله و شاهد تفاوت

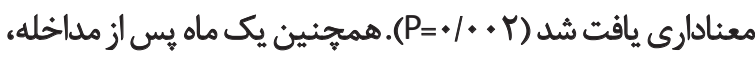

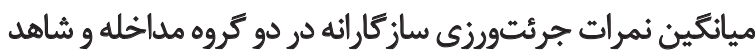

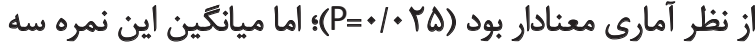

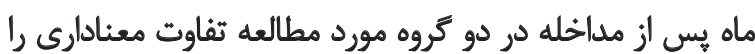

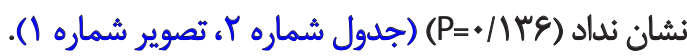

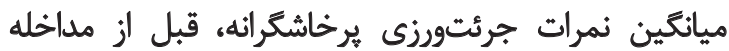

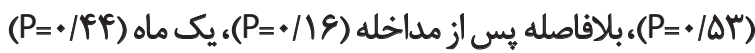

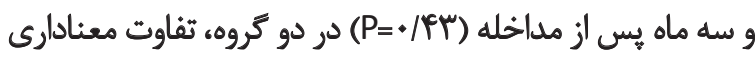

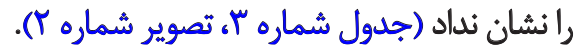
بر اساس آزمون آناليز واريانس با اندازههاي تكرارى بين

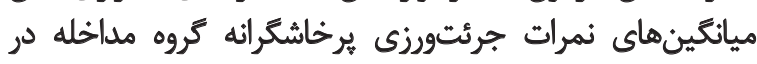

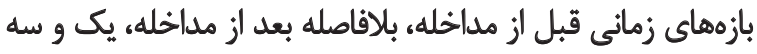




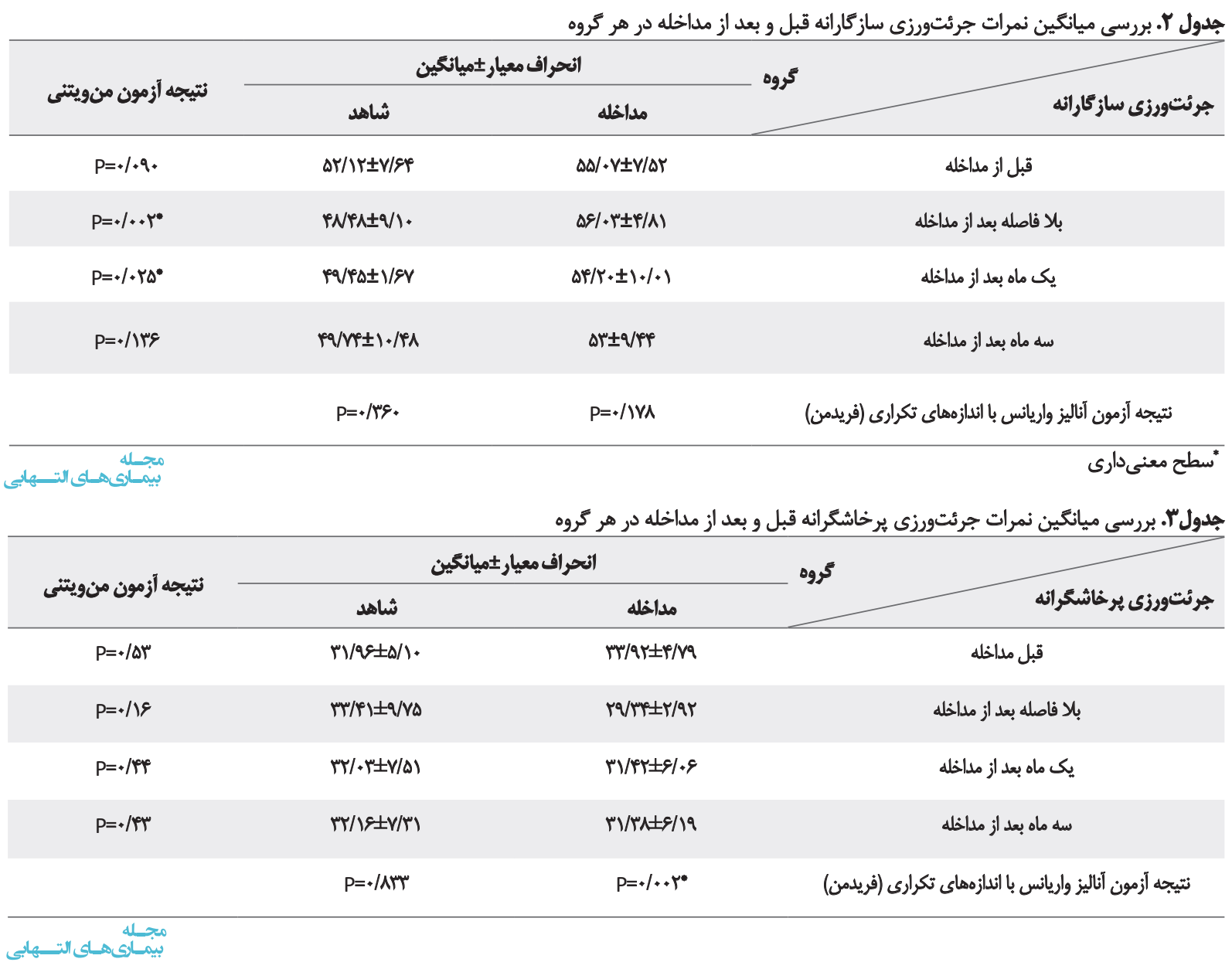

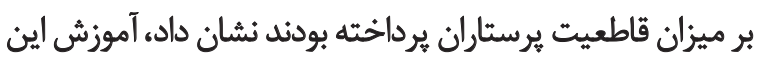

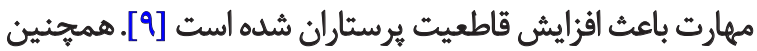

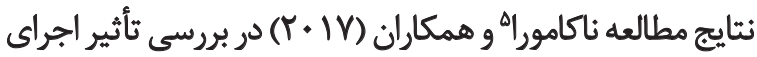

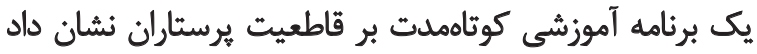

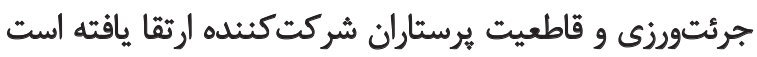

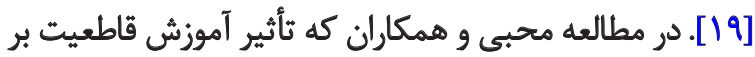

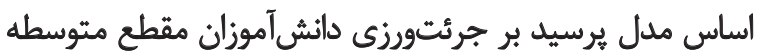

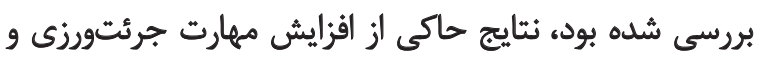

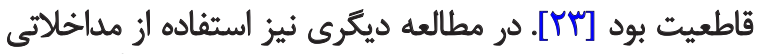

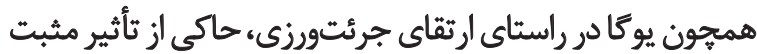

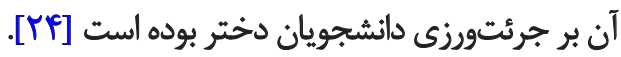

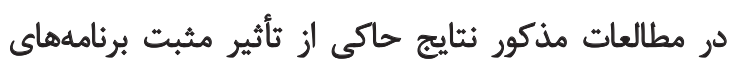

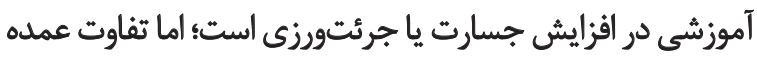

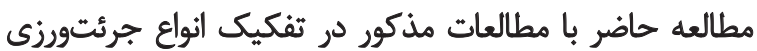

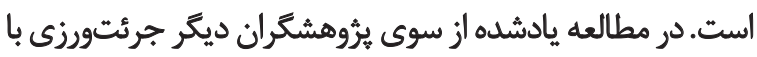

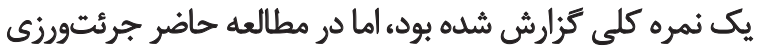

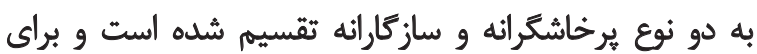

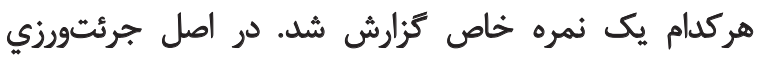

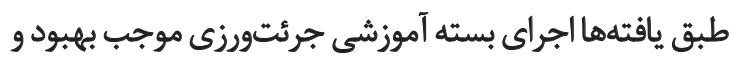

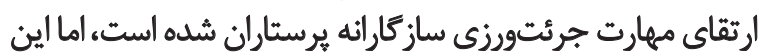

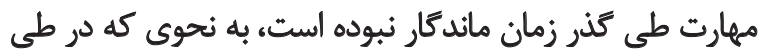

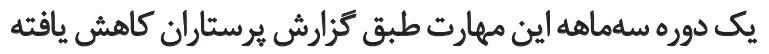

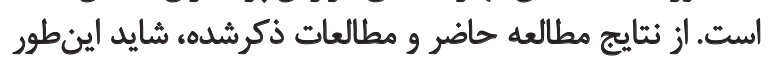

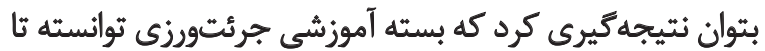

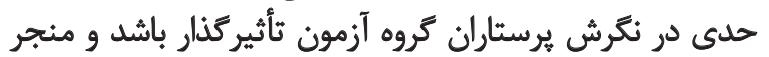

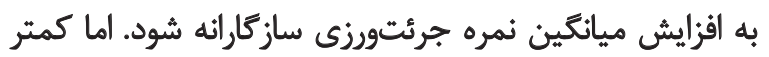

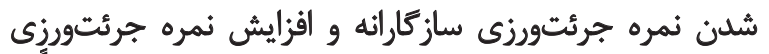

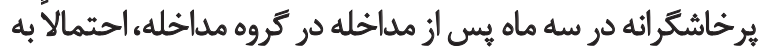

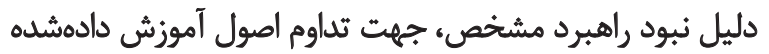

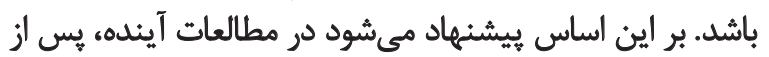
جلسات آموزشى، با هدف استمرار و تداوم يادئيرى ايجادشديد،

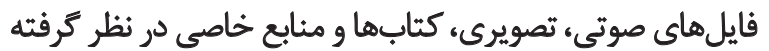

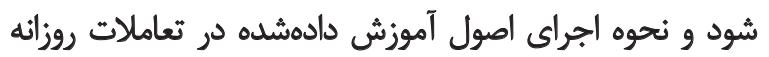
توسط مدرس دوره مورد تحليل و دوبارهسنجى قرائ دار كيرد.

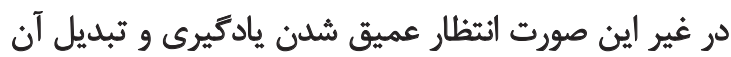

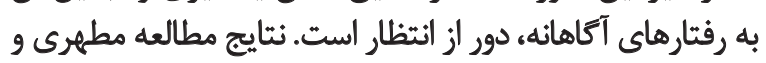

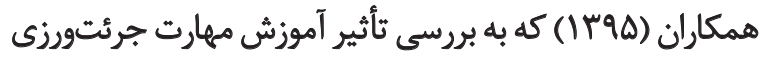




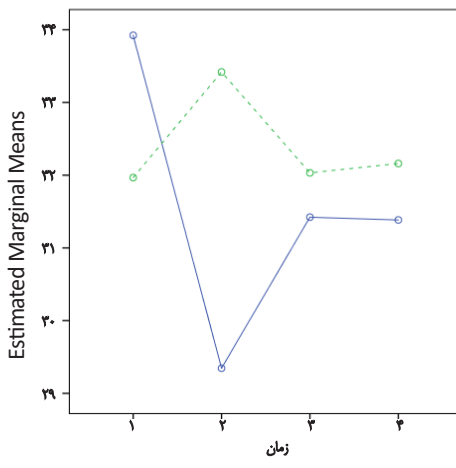

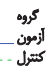

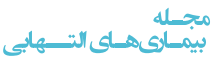

تصوير T. بررسى روند تغييرات ميانكين نمرات جراتورزى يرخاشكرانه در دو مروه آزمون وشاهد

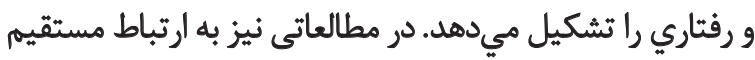

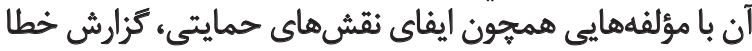

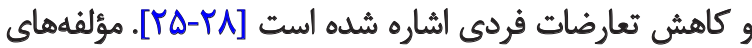

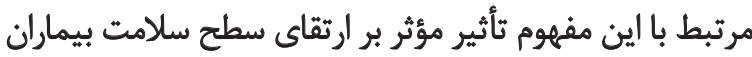

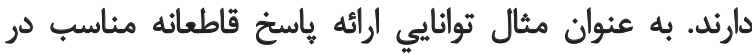

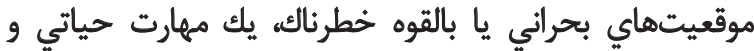

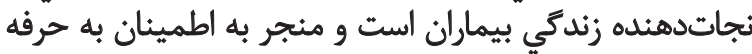

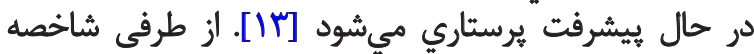

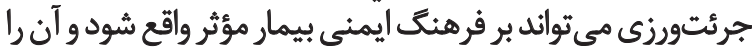

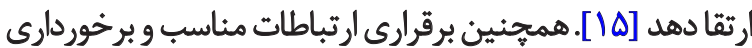

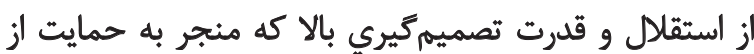

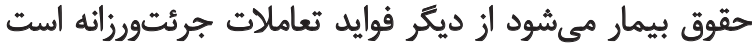

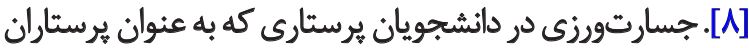

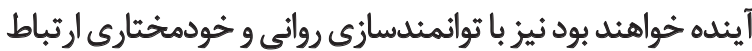

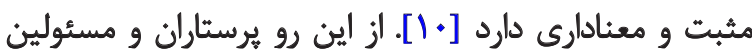

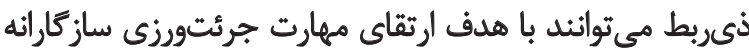

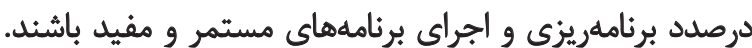

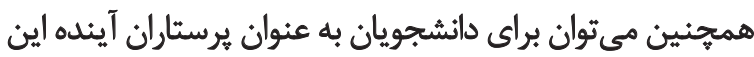

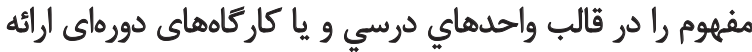

با توجه به اينكه تفاوت در مسائل فرهنكَى و اجتماعى، اعتقادات

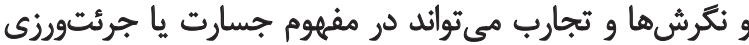

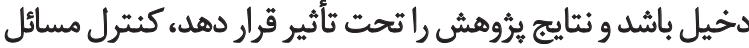

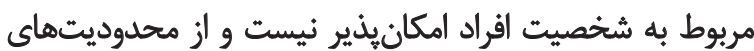

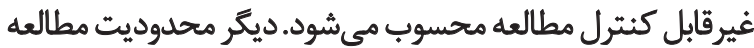

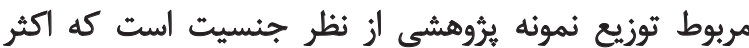

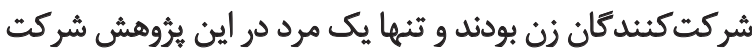

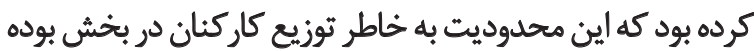

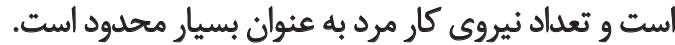
با توجه به اينكه جسارت بر دو نوع ساز مارانه و يرخاشكرانه

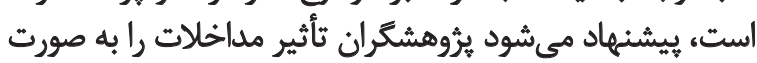

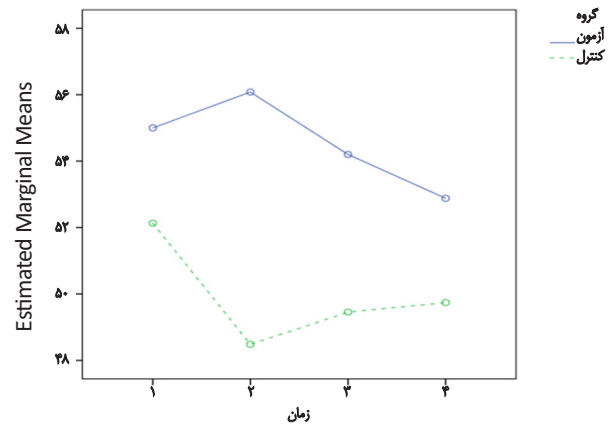

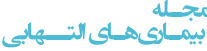

تصوير ا. بررسي روند ثغييرات ميانكين نمرات جراثورزى سازكاراثه در دو كروه آزمون و شاهد

سازكارانه نشاندهنده رفتارهاي فعالي است كه موجب ميشود

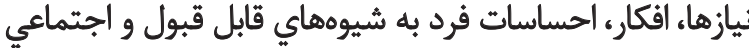

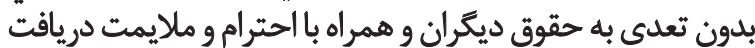

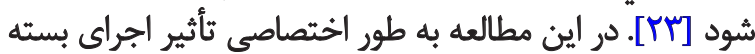

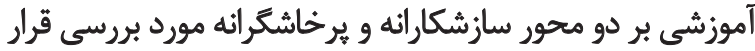

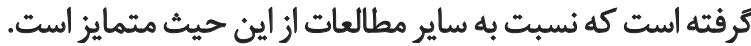

جرئتورزى يرخاشكرانه رفتارى مخرب و فعال محسوب

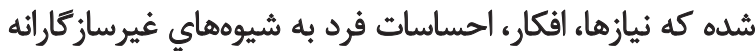

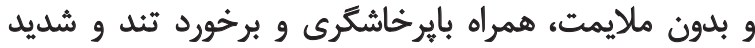

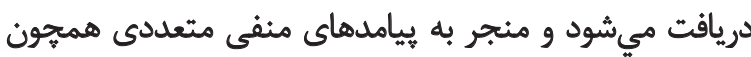

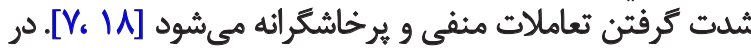

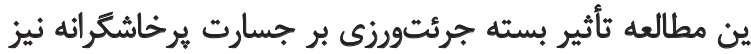

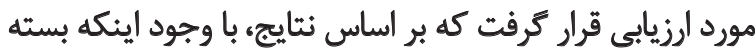

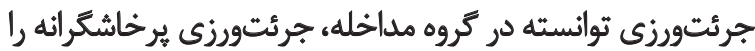

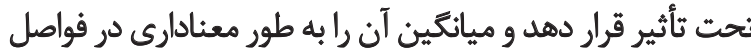

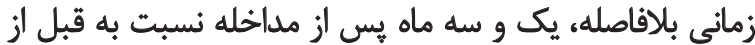

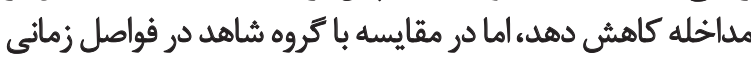

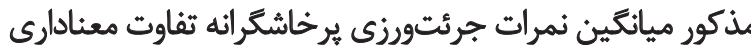

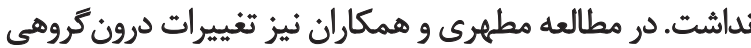

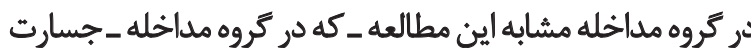

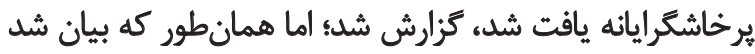

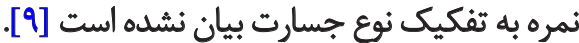
با توجه به اينكه تغيير رفتارهاي ناسازكارائه از ايجاد يك

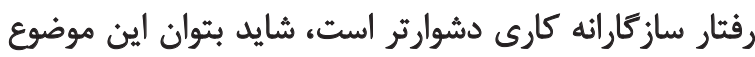

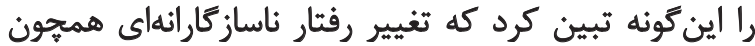

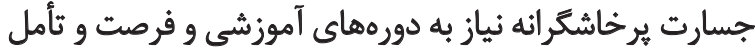

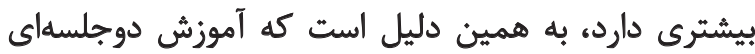
تفاوت معنادارى در دو كروه نداشته است.

قاطعيت يا ابراز وجود از مهمثرين و اساسيترين مهارتهاي

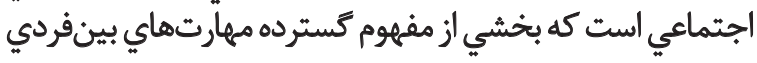


جداكانه روى هر دو مفهوم بسنجند. همجنين برانين بردي ارتباط

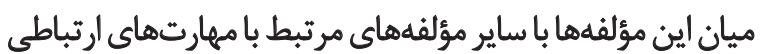

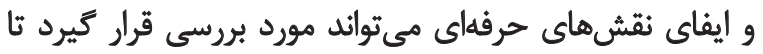

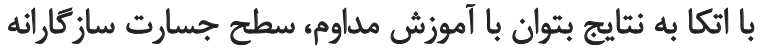

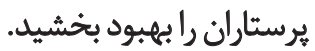

مالاحظاث الخالاقي

بيروى أز اصول اخلاق يثوهش

مطالعه حاضر در كميته اخلاق دانشكاه علوميزشكى قزئن

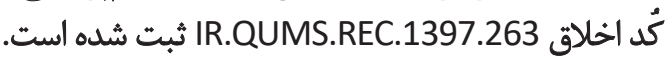

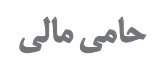

مقاله حاضر حاصل باياننامه كارشناسىارشد يرستارى نويسنده

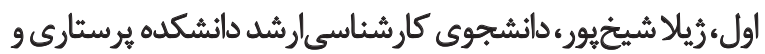
مامايى دانشعاه علوميزشكي قزوين استخ

$$
\text { مشاركت نويسندكان }
$$

نـعارش يرويـهوزال و محقـق اصلـى: زيـلا شـيخيور و سـيده

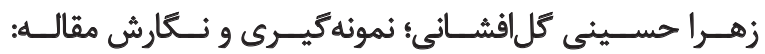

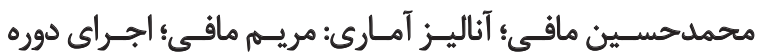

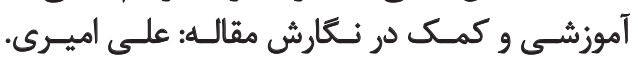

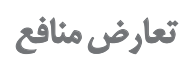

يُروهشكران اعلام مى دارئد هيج گونه تضاد منافعى در يثوهش

$$
\text { حاضر وجود ندارد. }
$$

تيم تحقيق با نهايت احترام مراتب تشكر خود را از همكارى

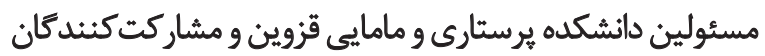

هحترم مطالعه اعلام مي دارد. 


\section{References}

[1] Yoshinaga N, Nakamura Y, Tanoue H, MacLiam F, Aoishi K, Shiraishi $Y$. Is modified brief assertiveness training for nurses effective? A single-group study with long-term follow-up. J Nurs Manag. 2018; 26(1):59-65. [DOI:10.1111/jonm.12521] [PMID]

[2] Seyyed Moharrami I, Pashib M, Tatari M, Mohammadi S. The efficiency of stress management group therapy in job stress and self-efficacy of nurses. J Torbat Heydariyeh Univ Med Sci. 2017; 5(1):42-9. [In Persian] http://jms.thums.ac.ir/article-1-406-en.html

[3] Mirzaei-Alavijeh M, Motlagh MI, Hosseini SN, Jalilian F. Nursing students communication skills with patients in Kermanshah University of Medical Sciences. Res Med Educ. 2017; 9(3):54-47. [In Persian] [DOI:10.29252/rme.9.3.54]

[4] Soni R, Kalaivani Srinivasan H. A pre- experimental study on the effect of 'Assertiveness training program' among nursing students of a selected college of Nursing, Ajitgarh, 2013-2014. Int J Appl Res. 2017; 3(5):533-8. https://www.allresearchjournal.com/ar chives $/$ ?year $=2017 \&$ vol=3\&issue $=5 \&$ part $=$ H\&Articleld $=3727$

[5] Ajibade BL, Adeleke MA, Adeolu E, Zacheus OO. Efficacy of assertiveness training in enhancing interpersonal skills among selected nurses attending MCPDP at Osun State School of Nursing, Osogbo: Osun State, Nigeria. J Health Med Nurs. 2015; 19:9-15. https://www.iiste.org/Journals/index.php/ JHMN/article/view/26747

[6] Ibrahim SAEA. Factors affecting assertiveness among student nurses. Nurse Educ Today. 2011; 31(4):356-60. [DOI:10.1016/j. nedt.2010.07.006] [PMID]

[7] Arslan E, Kılıç Akça N, Başer M. Levels of assertiveness and peer pressure of nursing students. Int J Caring Sci. 2013; 6(1):78-86. http://www.internationaljournalofcaringsciences. org/docs/10-ORIGINAL-PAPER-Vol-6-Issue-1.pdf

[8] Thrasher J, McNeely $\mathrm{H}$, Adrian B. When nursing assertion stops: A qualitative study to examine the cultural barriers involved in escalation of care in a pediatric hospital. Crit Care Nurs Clin North Am. 2017; 29(2):167-76. [DOI:10.1016/j. cnc.2017.01.004] [PMID]

[9] Motahari M, Mazlom SR, Asgharipour N, Maghsoudi Poor Zaid Abadi Sh, Ghiassi Ajgan M. The effect of the assertiveness skill training on the surface of nurse's aassertiveness in workplace. J Sabzevar Univ Med Sci. 2016; 23(4):644-51. [In Persian] http://jsums.medsab.ac.ir/article_894_en.html

[10] Azizi SM, Heidarzadi E, Soroush A, Janatolmakan M, Khatony AR. Investigation the correlation between psychological empowerment and assertiveness in nursing and midwifery students in Iran. Nurse Educ Pract. 2020; 42:102667. [DOI:10.1016/j.nepr.2019.102667] [PMID]

[11] Thompson RJ, Berenbaum H. Adaptive and Aggressive Assertiveness Scales (AAA-S). J Psychopathol Behav Assess. 2011; 33(3):323-34. [DOI:10.1007/s10862-011-9226-9]

[12] İlhan N, Sukut Ö, Akhan LU, Batmaz M. The effect of nurse education on the self-esteem and assertiveness of nursing students: A four-year longitudinal study. Nurse Educ Today. 2016; 39:72-8. [DOI:10.1016/j.nedt.2015.12.026] [PMID]
[13] Asi Karakaş S, Okanli A. The effect of assertiveness training on the mobbing that nurses experience. Workplace Health Saf. 2015; 63(10):446-51. [DOI:10.1177/2165079915591708] [PMID]

[14] Taghavi Larijani T, Sharifi Neiestanak ND, Aghajani M, Mehran A. Assertiveness and anxiety in midwifery \& nursing students. J Hayat. 2009; 15(2):61-72. [In Persian] http://hayat.tums.ac.ir/ article-1-121-en.html

[15] Rabani F, Kardan M, Wagharseyyedin SA, Bahrami M. Relationship between assertiveness and physician-nurse collaboration with safety culture. Q J Nurs Manag. 2019; 8(2):25-34. [In Persian] http://ijnv.ir/article-1-584-en.html

[16] Green J. Enhancing assertiveness in district nurse specialist practice. $\mathrm{Br}$ J Community Nurs. 2016; 21(8):400-3. [DOI:10.12968/bjcn.2016.21.8.400] [PMID]

[17] Yaghoobinejad N, Ahour T. The relationship between Iranian EFL students' personality characteristics and their assertiveness: A case of postgraduate students. J Engl Lang Pedagogy Pract. 2019; 12(24):137-55. [DOI:10.30495/JAL.2019.671930]

[18] Avşar F, Alkaya SA. The effectiveness of assertiveness training for school-aged children on bullying and assertiveness level. J Pediatr Nurs. 2017; 36:186-90. [DOI:10.1016/j. pedn.2017.06.020] [PMID]

[19] Nakamura Y, Yoshinaga N, Tanoue H, Kato S, Nakamura S, Aoishi K, et al. Development and evaluation of a modified brief assertiveness training for nurses in the workplace: A singlegroup feasibility study. BMC Nurs. 2017; 16:29. [DOI:10.1186/ s12912-017-0224-4] [PMID] [PMCID]

[20] Ahmed RA, Fakhry SF, Saad NF. Conflict resolution strategies training program and its effect on assertiveness among nursing students. Egypt J Health Care. 2019; 10(2):237-49. [DOI:10.21608/ejhc.2019.46255]

[21] Kim M. Development and effects of assertiveness training applying Dongsasub training for nursing students in clinical practice. J Korean Acad Nurs. 2016; 46(4):490-500. [In Korean] [DOI:10.4040/jkan.2016.46.4.490] [PMID]

[22] Moutabi F, Kazemzade Atufi M. Assertiveness behavior $5^{\text {t }}$ edition. Tehran: Miankoshk; 2012. [In Persian] http://opac. nlai.ir/opac-prod/bibliographic/2866903

[23] Khormayei F, Zare M. The relationship between family/ teachers' communication patterns and students' assertiveness through the mediating role of emotion regulation. Stud Learn Instr. 2017; 8(2):68-91. [In Persian] [DOI:10.22099/ JSLI.2017.3968

[24] Mohebi S, Sharifirad GHR, Shahsiah M, Botlani S, Matlabi M, Rezaeian M. The effect of assertiveness training on student's academic anxiety. J Pak Med Assoc. 2012; 62(3 Suppl 2):S37-41. [PMID]

[25] Karthika P. Influence of yoga intervention on assertiveness among under graduate students. Int J Physiol Nutr Phys Educ. 2020; 4(1):804-6. https://www.journalofsports.com/ pdf/2019/vol4issue1/PartR/5-1-81-530.pdf

[26] Imanifar N, Vaghar Seyedin SA, Roshanzadeh M, Afshar L. The relationship between patient advocacy practice of nurses and their assertiveness. Med Ethics. 2015; 9(32):141-66. [In Persian] [DOI:10.22037/mej.v9i32.9856] 
[27] Borimnejad L, Mardani Hmouleh M, Seyed Fatemi N, Mostaghaci $M$. Relationship between assertiveness and reporting of job errors among nurses. Q J Shahid Sadoughi Univ Med Sci (Tibbi-i-Kar). 2013; 5(2):60-5. [In Persian] http://tkj.ssu.ac.ir/ article-1-354-en.html

[28] Mazlom SR, Motahari M, Maghsoudi Poor Zaid Abadi Sh, Asgharipour N. Effect of assertiveness training on nurses' interpersonal conflicts. J Mazandaran Univ Med Sci. 2015; 25(124):107-18. [In Persian] http://jmums.mazums.ac.ir/article-1-5695-en.html 